\title{
Dynamic modelling to predict the likelihood of plant species persisting in fragmented landscapes in the face of climate change
}

\author{
$\underline{\text { M. Renton }}^{\text {a,b,c }}$, N. Shackelford ${ }^{\text {a,b }}$ and R. Standish ${ }^{\text {a,b,c }}$ \\ ${ }^{a}$ School of Plant Biology, The University of Western Australia \\ ${ }^{b}$ Western Australian Centre of Excellence for Climate Change, Forest and Woodland Health \\ ${ }^{c}$ CSIRO Ecosystem Sciences \\ Email: michael.renton@uwa.edu.au
}

\begin{abstract}
Many species are threatened by global climate change, but plants are particularly vulnerable because, as sessile organisms, they are unable to move to areas with more suitable conditions as the climate changes. Instead they must rely on their seeds dispersing far and often to keep pace with a changing climate. This problem is exacerbated by the fragmentation of natural landscapes by clearing for agricultural or urban development, or similarly by a species requirement for particular soil types or topography.
\end{abstract}

Models can help predict how different species will be affected by climate change. Most previous modelling work on predicting the persistence of plant and other species under climate change has been static, regression style modelling, known as climate envelope modelling. This has focussed on predicting where suitable environments for a species will likely occur under possible future climatic conditions, based on the species' distribution under current conditions. While the existence of suitable environments in a new climate is a necessary condition for a species' persistence, for sessile organisms such as plants, the ability of a species to move and colonise these suitable environments is also likely to be a major limitation. There is therefore a need for models that account for the dynamic processes involved in plant species' migration and colonisation in changing climates.

This paper presents such a dynamic model, called PPunCC (Plant Persistence under Climate Change). We describe how the PPunCC model represents the important factors and processes likely to affect a plant species' capacity to migrate across a landscape fast enough to keep pace with a changing climate, such as the rate of climate change, the degree of landscape fragmentation, and the plant species' life history, seed production, dispersal, and establishment. We also discuss how the model could be used to inform management decisions regarding adaptation options such as assisted migration or the creation of large-scale corridors that increase the connectivity of fragmented landscapes in order to help species migrate naturally and find suitable environments in new climates.

Keywords: spatially explicit, individual-based, dynamic model, dispersal, species distribution, functional traits, PPunCC 


\section{INTRODUCTION}

Many species are threatened by global climate change (Parmesan and Yohe, 2003). As sessile organisms, individual plants are unable to move to areas with more suitable climatic conditions as the climate changes and thus may be impacted to a greater degree than more mobile life forms. Instead, they must rely on their seeds dispersing far enough and quick enough to keep pace with a changing climate. This problem is exacerbated by the fragmentation of natural landscapes by clearing for agricultural or urban development, or similarly by a species requirement for particular soil types or topography.

The factors that affect a plant species' distribution across a landscape can be conceptualized as a series of filters (Krebs, 2001). Some parts of the landscape will be unsuitable for the plant species due to factors that will not vary with changing climate, such as soil, topography or clearing for agriculture or development. Other parts of the landscape will be unsuitable for the plant species due to climatic factors that will vary, such as average temperatures at different times of year, rainfall totals or annual patterns, evaporation or extreme events like short-term heat stress or frost. Other parts of the landscape will be unsuitable because of other factors that may depend on complex interactions between fixed factors and changing climate, such as the presence of pollinators to ensure fertilisation, the degree of stress caused by herbivores, competition with other plants and suitable conditions for germination. We can define a species' potential distribution as the full area it could potentially occupy under current conditions, as restricted by all the filters described above, and a species' realized distribution as that part of the potential distribution that the species has actually colonised. For plants with relatively specific requirements, or for any plant species in a fragmented landscape, the potential distribution will be a limited, fragmented and probably disconnected subset of the landscape; as climate changes, some parts of the potential distribution will disappear and new parts, which are likely to be disconnected from old parts, may appear. This means that in order to persist in the face of climate change, a species needs to not only have a potential distribution in the new climate, but also 'realise' at least some of this distribution by dynamically finding a path to it, in some cases by stepping over intervening unsuitable areas. For plants, without human intervention, this 'stepping' is almost certain to depend on the dispersal of seeds or possibly other propagules.

Various factors will affect not only the way the potential distribution of a species changes as the climate changes, but also the extent to which the species can dynamically realise this distribution. These include the species' functional ecological traits, such as plant life history (annual, short-lived perennial or long-lived tree), time to reach reproductive maturity, amount of seed production, dispersal mechanisms, rates of establishment, and range of environmental tolerance. These factors will also include environmental requirements of the species; rates and extent of climate change; and landscape characteristics such as availability of suitable niches and degrees and patterns of landscape fragmentation, and the availability of 'safe sites' within areas set aside for conservation and restoration.

Models can help predict how different species will be affected by climate change under different scenarios. These scenarios can include management options, so that models can help inform management decisions and policies. These models may attempt to take into account some or all of the factors discussed above. Much modelling to date, such as climate envelope modelling, has taken a relatively static and empirical approach, which predicts the new potential distribution of a species by extrapolating from characteristics of the current realized distribution, such as soil, topography and various climatic variables (e.g. Fitzpatrick et al., 2008; Yates, et al., 2010). This has meant that it has focussed on the total extent rather than the rate of climate change. This static approach is an important part of predicting the effects of climate change on species persistence, but does not take into account the equally important dynamic aspects.

Accounting for dynamic processes in models built to predict how different species will be affected by climate change is important. For example, a common criticism of climate envelope modelling is that it does not account for the dynamics of species migration. Studies based on climate envelope models have tended to predict the new potential distribution after a fixed change in the climate, and then consider the extremes of 'all or nothing' migration scenarios (the species can completely occupy its new potential distribution, or the species is restricted to the overlap between its new potential distribution and its old realized distribution) (Pearson and Dawson, 2003). Even when intermediate levels of migration are considered, this has tended to be simply an estimated maximum speed of migration. The migration ability of a species will actually depend on functional traits such as its life history, fecundity, dispersal and establishment traits. Current fragmentation, land use patterns, and variability in soil type, together with the rate of climate change, are also likely to interact with functional traits of species to affect their ability to migrate and survive climate change by colonising their new potential distribution. 
To address this issue, we developed a spatially-explicit dynamic model, called PPunCC (Plant Persistence under Climate Change), which focuses on landscape fragmentation and the dynamic aspects of climate change, plant life history, seed production, dispersal, and establishment. The purpose of this model is to enable studies to help make predictions to address questions such as:

- what kind of plant species, with which kind of functional trait combinations, will be most likely to survive climate change in different landscapes,

- which traits, landscape characteristics and aspects of climate change will have the greatest impact on a species' chances of surviving global climate change (GCC),

- how will strategic restoration of plant habitat, to increase landscape connectivity and create corridors, affect the probability of persistence of species with different functional ecological traits.

\section{PPUNCC MODEL}

\subsection{Overview}

The PPunCC model simulates the age-structured population dynamics and dispersal of a single plant species across a grid-based landscape. Based on an annual time step, the model simulates population dynamics over several centuries. The model tracks the number of plants and seeds in each cell as well as the age of each plant. PPunCC model parameters are listed and described in Table 1. The model is implemented in the $\mathrm{R}$ programming language and is freely available upon request to the authors.

\subsection{Landscape and climate}

The landscape is represented as a grid of square cells, each of which is either suitable or unsuitable as habitat for the plant species (Figure 1). The fragmentation proportion of the landscape, $p_{f}$, is defined as the proportion of cells that are unsuitable habitat for plant growth. Unsuitability could represent any type of abiotic or biotic filter. Landscapes can either be wholly specified by the user or randomly generated. If randomly generated, the full landscape starts as suitable and then cells are randomly selected and converted to being unsuitable until the proportion of unsuitable cells equals $p_{f}$. Cell selection can be dependent or independent of previous selection, to generate spatial patterns that are clustered, random or regular. Every cell is assigned a value $c_{c e l l}$ representing the climatic variable of interest in an individual cell, which changes at a rate of $r_{c c} \mathrm{yr}^{-1}$ during periods of climate change. This variable can represent any particularly critical climate variable, such as average total annual rainfall or maximum summer temperatures, or a combination of such variables.

For the illustrative examples in this paper, grid

Table 1. Parameters of the PPunCC model with description.

\begin{tabular}{|c|c|}
\hline \multicolumn{2}{|r|}{ Landscape parameters } \\
\hline$p_{f}$ & Proportion of landscape that is unsuitable habitat \\
\hline$c_{\text {cell }}$ & Value of climatic variable of interest in individual cell \\
\hline$r_{c c}$ & Rate of climatic change (per year) \\
\hline$\overline{D_{\text {cell }}}$ & Number of plants present in cell \\
\hline \multicolumn{2}{|r|}{ Plant functional trait parameters } \\
\hline$F$ & $\begin{array}{l}\text { Effective fecundity: } F=N_{s} \times p_{s} \text { (where } N_{s} \text { is the number of seeds } \\
\text { produced by each mature plant each year and } p_{s} \text { is the seed } \\
\text { establishment probability in optimal climatic conditions) }\end{array}$ \\
\hline$D$ & Maximum plant density \\
\hline$T$ & Threshold dispersal distance \\
\hline$p_{m}$ & Probability of mortality \\
\hline$Y_{m}$ & Years to reproductive maturity \\
\hline$k$ & Exponential dispersal parameter \\
\hline$\lambda$ & Leptokurtic dispersal parameter \\
\hline \multicolumn{2}{|r|}{ Plant climatic response parameters } \\
\hline$c_{\text {opt }}$ & Plant optimal climatic value \\
\hline$c_{t o l}$ & Plant climatic tolerance \\
\hline$p_{e}$ & $\begin{array}{l}\text { Probability of seedling establishment in specific climatic } \\
\text { conditions }\end{array}$ \\
\hline
\end{tabular}
cells are defined to be a square kilometre in area, and the full grid is 100 cells by 20 cells, representing an area $100 \mathrm{~km}$ long in the east-west direction by $20 \mathrm{~km}$ wide in the north-south direction. The climatic variable represents annual rainfall, defined to vary linearly in the east-west direction along the $100 \mathrm{~km}$ length of the area and to be constant in the north-south direction. We initialised the simulations with an annual rainfall of $500 \mathrm{~mm} \mathrm{yr}^{-1}$ at the eastern end and $800 \mathrm{~mm} \mathrm{yr}^{-1}$ at the western end. Rainfall was kept at these levels for a period at the start of the simulations, to allow the distribution of the species to stabilise, and was then

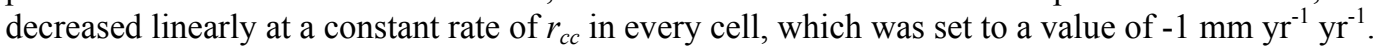




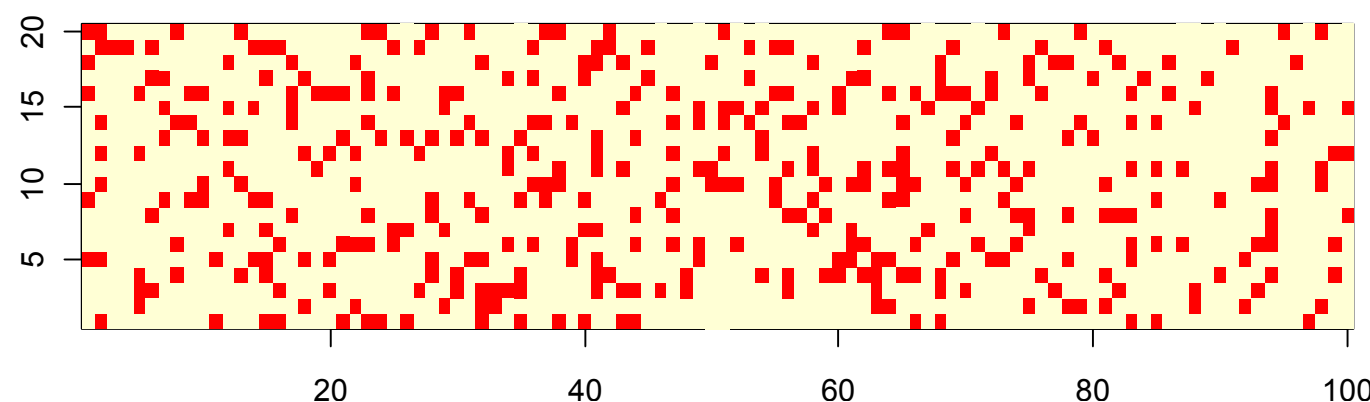

Figure 1. An example landscape consisting of suitable (red) and unsuitable (light yellow) grid cells (i.e., plant habitat). Rainfall is modelled as a linear gradient from dry in the east to wet in the west. Note that in this example, $p_{f}=0.8$, so $80 \%$ of the landscape is unsuitable habitat for plant growth.

\subsection{Fecundity and life cycle}

The PPUnCC model contains a number of parameters representing species' functional characteristics. $N_{s}$ is the number of seeds produced by each mature plant each year and $p_{s}$ is the probability, in optimal climatic conditions, that each individual seed will establish by dispersing to a suitable microhabitat, germinating and surviving the first year. To simplify the analysis, we synthesised these two parameters in another parameter $F$ which is defined as $F=N_{s} \times p_{s}$. This parameter $F$ represents the 'effective fecundity', or the number of seeds produced per plant per year that will germinate and establish (survive to the end of their first year) in optimal climatic conditions. Plant density $(D)$ is the maximum carrying capacity of the landscape, or specifically the number of plants per hectare that could establish in optimal climatic conditions, taking into account the number of suitable microhabitats. It can thus be seen as a measure of the rarity/commonness of the species at a local-scale in usual conditions without accounting for climate change. Probability of mortality $\left(p_{m}\right)$ is the annual probability of mortality of established plants and is assumed to be constant. Years to maturity $\left(Y_{m}\right)$ is the age at which a plant produces seeds for the first time.

\subsection{Plant establishment and the effect of climate}

A species is assumed to have a preferred value for the climatic variable, $c_{\text {opt }}$, which in this case represents the annual rainfall amount at which its growth is maximised. It is also assumed to have a degree of tolerance to variation in this variable, which is captured in the parameter $c_{t o l}$. Seedling establishment is a key point of vulnerability to climatic factors for the plants (Grubb, 1977; Harper, 1977), and thus the effect of climate is modelled as a factor that scales the probability of establishment in an optimal climate, $p_{s}$. The probability of establishment $p_{e}$ of a seedling from seed in a specific cell with a specific climate $c_{c e l l}$ is thus assumed to depend on the equation:

$$
p_{e}=\frac{1}{1+\left(\frac{c_{c e l l}-c_{o p t}}{c_{\text {tol }}}\right)^{2}} * p_{s}
$$

This means that $p_{e}$ is equal to $p_{s}$ when climate is optimal $\left(c_{c e l l}=c_{\text {opt }}\right)$, decreases smoothly as climate increases or decreases from optimal until $p_{e}$ is equal to one-half $p_{s}$ when $c_{c e l l}=c_{o p t} c_{\text {tol }}$, and tends towards zero as the difference between $c_{c e l l}$ and $c_{\text {opt }}$ increases beyond $c_{\text {tol }}$ (Fig. 2). In other words, $c_{t o l}$ is the difference from optimal climate (the difference from optimal annual rainfall in this case study) at which the probability of establishment falls to half of what it is in the optimal climate $c_{\text {opt }}$. For our examples, $c_{\text {opt }}$ was set to $510 \mathrm{~mm}$ $\mathrm{yr}^{-1}$, meaning the optimal area for the species was initially in the dry (far east) end of the modelled landscape, and $c_{t o l}$ was set to 3 , meaning that only a small section of the landscape $-6 \mathrm{~km}$ from east to west - had a relatively favourable climate for the species (Figure 2).

Establishment is also limited by the maximum plant density $D$. The model tracks the number of plants in an individual cell at each time-step, $D_{\text {cell }}$, and germination is constrained to be no more than $D_{\text {cell }}-D$. Therefore, at any time within an individual cell, the number of plants present cannot exceed the maximum, $D$. While we chose to model establishment as the primary limitation to species persistence, the model could be easily adapted to include changes in seed production or mortality rates of established plants, if this was deemed to be important for a particular case study. 


\subsection{Dispersal}

Seed dispersal is modelled using a kernel or probability distribution, an empirical approach commonly used to represent the various different processes involved in seed dispersal in a relatively simple way (Kot et al. 1996). Threshold dispersal distance $(T)$ is a parameter that defines the distribution of dispersal distances; it is the threshold distance beyond which we would expect to find exactly one percent of seeds dispersing, or equivalently, $99 \%$ of seeds will fall within this distance. As a default, we assume that dispersal follows an exponential distribution, with a cumulative distribution function (cdf)

$$
p(d ; k)=1-e^{-k d}
$$

where $p$ is the probability of a seed dispersing less than $d$ meters from the maternal plant, and thus from the definition of $T$ above, it follows that

$$
k=\frac{\log (100)}{T} .
$$

Seed dispersal is simulated stochastically. Conceptually, the dispersal for each seed is simulated individually, with distance of dispersal generated using the dispersal kernel and the direction of dispersal drawn from a uniform distribution. Seeds are assumed to disperse from the centre of the cell in which they are produced. A version of the model based directly on this conceptual procedure was used for early testing, but for computational efficiency, another equivalent algorithm was developed. This more computationally efficient algorithm works as follows. At the start of each simulation run, the dispersal kernel is integrated across a large grid for a single source cell in the centre of the grid. Because the model is based on a regular grid, and we assume the dispersal kernel is independent of landscape details, this gives us a matrix $M$ containing the probabilities that a seed originating from a central source cell will

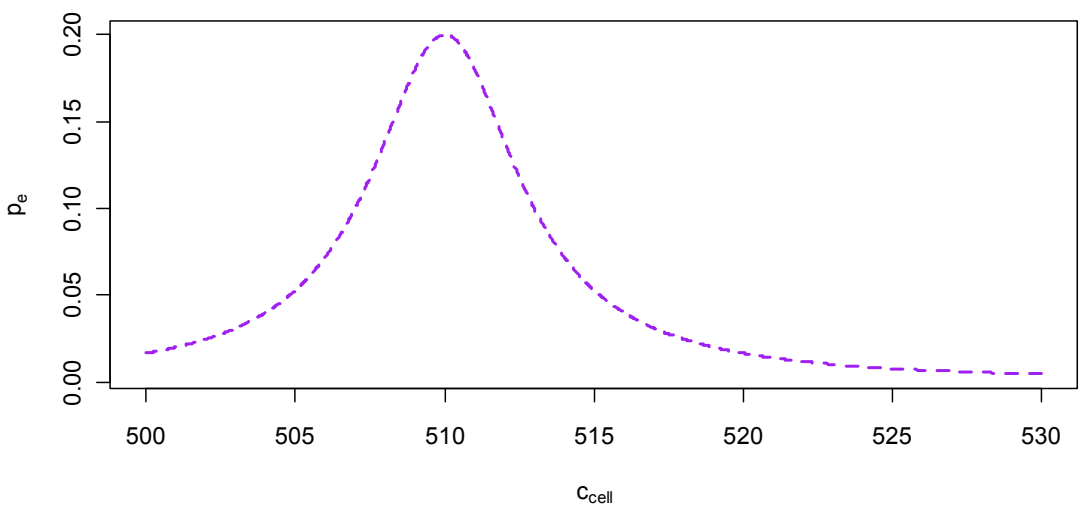

Figure 2. Probability of establishment $p_{e}$ varying with climate within a cell $c_{\text {cell }}$

$$
\text { when } p_{s}=0.2, c_{\text {opt }}=510 \text { and } c_{\text {tol }}=3 \text {. }
$$
disperse to any particular destination cell. When the simulation is run, at each time step all possible combinations of source and destination cells are considered in turn, and the actual number of seeds travelling from a source cell to a destination cell is drawn from a binomial distribution with $n$ equal to the number of seeds produced in the source cell in that time step, and $p$ obtained from the matrix $M$. Initial testing showed that the computationally efficient algorithm was equivalent to the conceptual procedure. Moreover, the size of $M$ could be reduced to the point where it covered only the area of the landscape that was relatively climactically suitable.

In order to test the sensitivity of the results to a change in the functional form of the dispersal kernel, we also implemented an alternative fat-tailed (leptokurtic) distribution, because seed dispersal patterns are generally well described by this function (Howe and Smallwood, 1982). The fat-tailed alternative we considered was the Weibull distribution with shape parameter equal to 0.5 and thus a cumulative distribution function

$$
p(d ; \lambda)=1-e^{-\sqrt{d / \lambda}}
$$

where $p$ is the probability of a seed dispersing less than $d$ meters from the maternal plant, and thus from the definition of $T$ above, it follows that

$$
\lambda=\frac{T}{(\log (100))^{2}}
$$




\subsection{Model dynamics}

The simulation proceeds on an annual time step. All seeds in a grid cell germinate at the start of a growing season; we assume no dormant soil seed bank. A proportion $p_{e}$ of these seedlings establish, based on the climatic suitability of the cell $c_{\text {cell }}$ and limited by the maximum plant density $D$, and are added to the plant population as year-old plants. No seedlings establish in unsuitable grid cells. Each mature plant (age $\left.\geq Y_{m}\right)$ then produces $N_{s}$ seeds, which disperse as described above based on the dispersal parameter $T$, and are assumed to arrive in a suitable microhabitat within their destination cell with probability $p_{s}$. Seeds that arrive in an unsuitable cell or do not find a suitable microhabitat are assumed to die. At the end of the growing season the age of all plants is incremented by one year.

The simulation is consistently initialised with a small number of mature plants in every suitable grid cell, and then run for 40 years with a constant climate to allow the spatial distribution as well as the age distribution of the population to stabilise. This simulation results in extinction of plants in cells with unsuitable climates and a stable population in cells with suitable climates. At this point climate change is commenced and the spatial distribution of the plant species changes in response to this climate change, with the species either following the suitable environment across the landscape, or becoming regionally extinct.

\section{EXAMPLE OUTPUTS}

The base output of a model run is simply whether the species persist for the full period of climate change or not. Since the model is stochastic, it can be run a number of times to derive a probability of persisting for a given set of parameter values. If desired, animated visualisation of model dynamics can be obtained (Figure 3 ) in order to track the process of a species migrating across a landscape under climate change.
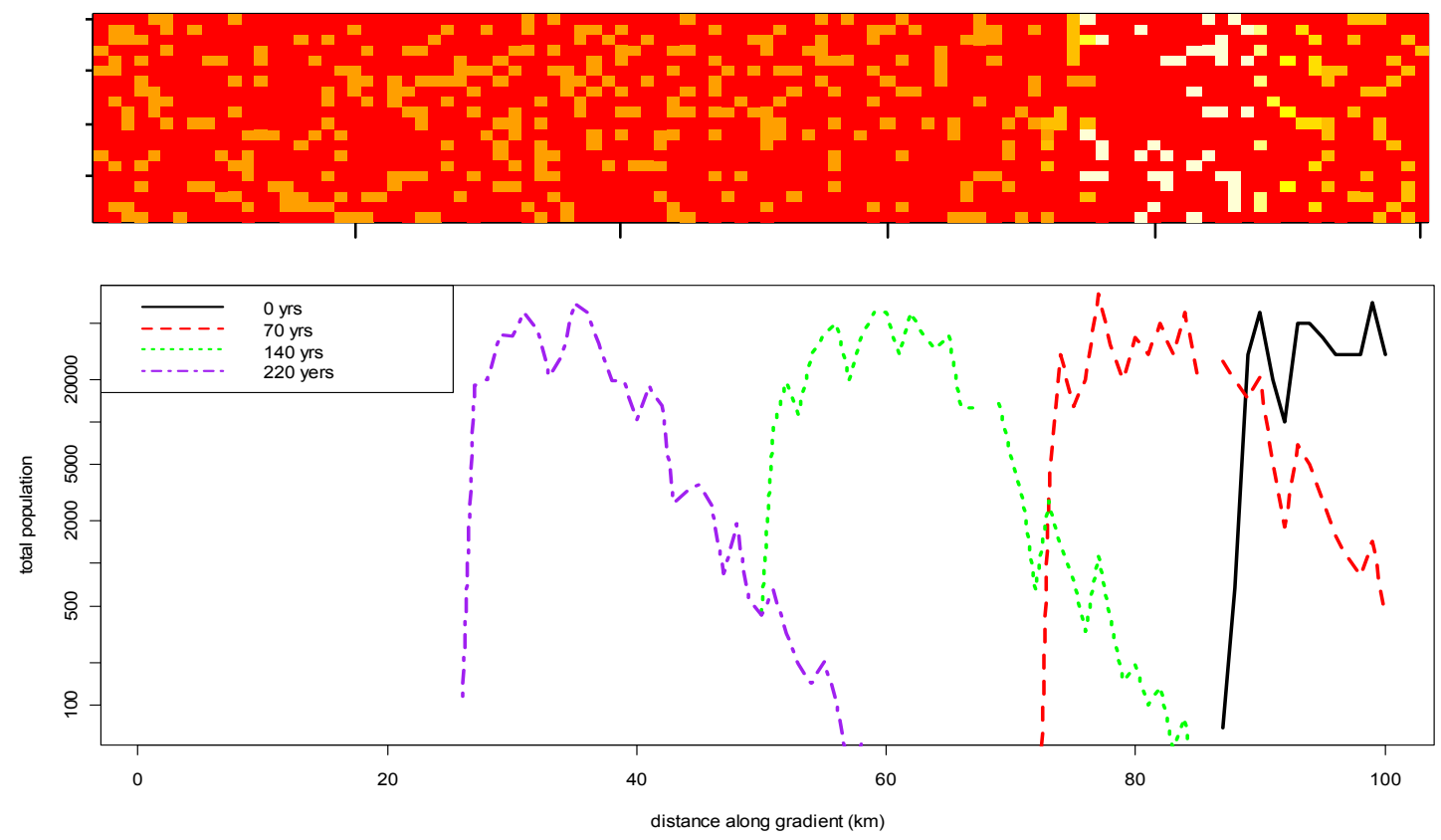

Figure 3. Model output from an example run with $80 \%$ fragmentation and an annual plant with high fecundity and dispersal. The top plot shows the population density in different grid cells 40 years after climate change began: lighter cells correspond to greater density and the darkest cells indicate unsuitable environments. The bottom plot shows the total population summed across the gradient at different distances along the gradient at $0,70,140$ and 220 years after climate change began.

\section{DISCUSSION AND CONCLUSIONS}

We plan to use the PPunCC model to investigate what kinds of plant species, with which kind of functional trait combinations, will be most likely to survive climate change in different landscapes. A large set of model runs will be conducted over a large set of combinations of model parameter values, with a number of replicates for each combination. These results will allow us to draw conclusions on which species are most 
likely to persist in the face of a changing climate. For selected 'species', as represented by particular combinations of functional trait parameters, we will conduct further sensitivity analyses to investigate which traits, landscape characteristics and aspects of climate change will have the greatest impact on the species' chances of surviving climate change. We will also conduct simulations in landscapes with different degrees of fragmentation, to look at how this affects results. Finally we will simulate targeted restoration of plant habitat by changing unsuitable cells to suitable at specific locations within landscapes chosen to increase landscape connectivity and create corridors, to determine how this will affect the probability of different functional plant types persisting.

The static aspects of environmental suitability, such as soil and landscape use, and the dynamic aspects of climatic suitability are represented in a relatively abstract way in the current version of PPunCC. This is because the focus of the investigation will be on drawing general conclusions valid across many species, landscapes and situations, rather than trying to calibrate the model to accurately represent a particular species, landscape or situation. However, for a more specific application, a separate bioclimate envelope style model (Pearson and Dawson, 2003) based on output of established climate change prediction models, together with empirical data on relevant environmental characteristics across the landscape and the current distribution of a species, could be used to provide realistic maps of the locations predicted to be both environmentally and climatically suitable for the species each year. These maps could then be used as the landscape suitability maps on which PPunCC bases its predictions. This would allow us to start to more realistically account for the complex interactions between the characteristics of particular landscapes and plant species, multiple aspects of local-scale climate change and dynamic biological processes in predicting outcomes for particular species in particular landscapes.

The results obtained from the model, whether generalised or more specific, will give a prediction of what kind of functional types are likely to be able to persist without human intervention in different landscapes under various climate change scenarios. They will also help predict what kind of functional types are likely to be able to persist with various levels of targeted (or untargeted) habitat restoration aimed at creating largescale corridors that increase the connectivity of fragmented landscapes and allow species to migrate to suitable environments in new climates, and thus help decide whether such interventions are worthwhile. Finally, they will help identify what species are unlikely to persist with the planned levels of restoration in the landscape of interest, and thus require interventions such as 'assisted migration' to help them find suitable environments in new climates.

\section{ACKNOWLEDGMENTS}

Michael acknowledges support of the Western Australian State Government in funding the Centre of Excellence for Climate Change, Forest and Woodland Health.

\section{REFERENCES}

Fitzpatrick, M., Gove, A., Sanders, N. and Dunn, R. (2008). Climate change, plant migration, and range collapse in a global biodiversity hotspot: the Banksia (Proteaceae) of Western Australia. Global Change Biology, 14(6), 1337-1352.

Grubb, P.J. (1977). The maintenance of species-richness in plant communities: the importance of the regeneration niche. Biological. Reviews, 52(1), 107-145.

Harper, J.L. (1977). Population Biology of Plants. Academic Press.

Howe, H.F. and Smallwood, J. (1982). Ecology of seed dispersal. Annual Review of Ecology and Systematics, 13: 201-228.

Kot, M., Lewis, M.A. and van den Driessche, P. (1996) Dispersal Data and the Spread of Invading Organisms. Ecology. 77(7):2027.

Krebs, C.J. (2001). Ecology (5th ed.): Benjamin/Cummings Publishing Company.

Parmesan, C., and Yohe, G. (2003). A globally coherent fingerprint of climate change impacts across natural systems. Nature, 421(6918), 37-42.

Pearson, R., and Dawson, T. (2003). Predicting the impacts of climate change on the distribution of species: are bioclimate envelope models useful? Global Ecology and Biogeography, 12(5), 361-371.

Yates, C., McNeill, A., Elith, J. and Midgely, G. (2010). Assessing the impacts of climate change and land transformation on Banksia in the South West Australian Floristic Region. Diversity and Distributions, 16(1), 187-201. 